\title{
INEQUALITIES FOR CONVEX FUNCTIONS
}

\author{
HAJRUDIN FEJZIĆ AND FUAD ŽIVOJEVIĆ
}

\begin{abstract}
We consider inequalities of the form $\sum_{i=0}^{m} a_{i} \varphi\left(b_{i}\right) \geq 0$, and we give necessary and sufficient conditions on the nodes $b_{0}, b_{1}, \ldots, b_{m}$, and the weights $a_{i}$ for such an inequality to be true for every real convex function $\varphi$. In the case the nodes are integers with $b_{0}$ the smallest of them, then $\sum_{i=0}^{m} a_{i} \varphi\left(b_{i}\right) \geq 0$ if and only if $x^{-b_{0}} \sum_{i=0}^{m} a_{i} x^{b_{i}} /(x-1)^{2}$ is a polynomial with positive coefficients.
\end{abstract}

\section{INTRODUCTION}

A real function $\varphi$ is convex if and only if $\frac{\varphi(v)-\varphi(u)}{v-u} \leq \frac{\varphi(w)-\varphi(v)}{w-v}$ whenever $u<v<w$ are in its domain. The last inequality can be replaced by the equivalent form

$$
(w-v) \varphi(u)+(u-w) \varphi(v)+(v-u) \varphi(w) \geq 0 .
$$

Convex functions are extremely useful in proving inequalities mainly because of Jensen's inequality, a finite form of which states that if $\varphi$ is a real convex function, if the numbers $x_{1}, x_{2}, \ldots, x_{n}$ are in its domain, if the weights $a_{i}$ are positive, then $\varphi\left(\frac{\sum_{i=1}^{n} a_{i} x_{i}}{\sum_{i=1}^{n} a_{j}}\right) \leq \frac{\sum_{i=1}^{n} a_{i} \varphi\left(x_{i}\right)}{\sum_{i=1}^{n} a_{j}}$. Another inequality for convex functions is the so called Karamata's inequality. Let $x_{1} \leq x_{2} \leq \cdots \leq x_{n}$ and $y_{1} \leq y_{2} \leq \cdots \leq y_{n}$ be in the domain of a convex function $\varphi$. Suppose that $\sum_{i=k}^{n} y_{i} \leq \sum_{i=k}^{n} x_{i}$ for $k=2,3, \ldots, n$ and that $\sum_{i=1}^{n} y_{i}=\sum_{i=1}^{n} x_{i}$. Then Karamata's inequality is that $\sum_{i=1}^{n} \varphi\left(x_{i}\right) \geq$ $\sum_{i=1}^{n} \varphi\left(y_{i}\right)$. The reader can find further information on Karamata's and related inequalities in [1], which contains an extensive bibliography on the subject.

The conclusions of Jensen's and Karamata's inequalities are of the form $\sum_{i=0}^{m} a_{i} \varphi\left(b_{i}\right) \geq 0$ for every appropriate convex function. Because 1 and -1 are convex, $\sum_{i=0}^{m} a_{i}=0$ and because $x$ and $-x$ are convex, $\sum_{i=0}^{m} a_{i} b_{i}=0$.

2010 Mathematics Subject Classification. Primary: 26A24.

Key words and phrases. Convex functions, inequalities. 
In this paper we show that such an inequality is true for every real convex function $\varphi$ provided it holds for the $m+1$ convex functions

$$
g_{k}(x)=\left\{\begin{array}{ll}
0 & x<b_{k} \\
x-b_{k} & x \geq b_{k}
\end{array} .\right.
$$

Then we use that result to prove Jensen's and Karamata's inequalities. Next we present a simple characterization when the nodes are integers (or if the spacing between nodes are integer multiples of some $h$ ). The result in this case is that $\sum_{i=0}^{m} a_{i} \varphi\left(b_{i}\right) \geq 0$ for every real convex function if and only if $x^{-b_{0}} \sum_{i=0}^{m} a_{i} x^{b_{i}} /(x-1)^{2}$ is a polynomial with positive coefficients. Two examples are given to illustrate the "effectiveness" of this characterization. We finish the paper with a brief discussion of inequalities for $n$ convex functions.

\section{NeCESSARY AND SUfFicient CONDITIONS}

If $f$ is a function, then we denote by $[f: u, v, w]$ the operator

$$
[f: u, v, w]=(w-v) f(u)+(u-w) f(v)+(v-u) f(w) .
$$

From (1) it follows that $\varphi$ is convex if and only if $[\varphi: u, v, w] \geq 0$ whenever $u<v<w$ are in its domain. One obvious property of this operator is that $[c x+d: u, v, w]=0$. This property will be used in the proof of Theorem 2 below.

Proposition 1. If $\sum_{i=0}^{m} a_{i}=0$ and $\sum_{i=0}^{m} a_{i} b_{i}=0$ where $b_{0}<b_{1}<\cdots<b_{m}$, then there are numbers $\alpha_{0}, \ldots, \alpha_{m-2}$ such that for every function $f$ we have $\sum_{i=0}^{m} a_{i} f\left(b_{i}\right)=\sum_{j=0}^{m-2} \alpha_{j}\left[f: b_{j}, b_{j+1}, b_{j+2}\right]$.

Proof. Let $\alpha_{0}=a_{0} /\left(b_{2}-b_{1}\right)$; then we can write $\sum_{i=0}^{m} a_{i} f\left(b_{i}\right)-\alpha_{0}[f$ : $\left.b_{0}, b_{1}, b_{2}\right]$ as $\sum_{i=1}^{m} a_{i}^{\prime} f\left(b_{i}\right)$. Notice that $\sum_{i=1}^{m} a_{i}^{\prime}=\sum_{i=0}^{m} a_{i}-\alpha_{0}\left(\left(b_{2}-b_{1}\right)+\right.$ $\left.\left(b_{0}-b_{2}\right)+\left(b_{1}-b_{0}\right)\right)=0$ and similarly $\sum_{i=1}^{m} a_{i}^{\prime} b_{i}=\sum_{i=0}^{m} a_{i} b_{i}-\alpha_{0}\left(\left(b_{2}-\right.\right.$ $\left.\left.b_{1}\right) b_{0}+\left(b_{0}-b_{2}\right) b_{1}+\left(b_{1}-b_{0}\right) b_{2}\right)=0$. Next let $\alpha_{1}=a_{1}^{\prime} /\left(b_{3}-b_{2}\right)$; then

$$
\begin{aligned}
\sum_{i=0}^{m} a_{i} f\left(b_{i}\right)-\alpha_{0}\left[f: b_{0}, b_{1}, b_{2}\right] & -\alpha_{1}\left[f: b_{1}, b_{2}, b_{3}\right] \\
= & \sum_{i=1}^{m} a_{i}^{\prime} f\left(b_{i}\right)-\alpha_{1}\left[f: b_{1}, b_{2}, b_{3}\right]=\sum_{i=2}^{m} a_{i}^{\prime \prime} f\left(b_{i}\right)
\end{aligned}
$$

with $\sum_{i=2}^{m} a_{i}^{\prime \prime}=0$ and $\sum_{i=2}^{m} a_{i}^{\prime \prime} b_{i}=0$. Continuing this way we obtain $\sum_{i=0}^{m} a_{i} f\left(b_{i}\right)-\sum_{j=0}^{m-2} \alpha_{j}\left[f: b_{j}, b_{j+1}, b_{j+2}\right]=c f\left(b_{m-1}\right)+d f\left(b_{m}\right)$ with $c+d=0$ and $c b_{m-1}+d b_{m}=0$. But $c+d=0$ and $c b_{m-1}+d b_{m}=0$ if and only if $c=d=0$.

Now we are ready to prove our main result. 
Theorem 2. Suppose that $\sum_{i=0}^{m} a_{i}=0$ and $\sum_{i=0}^{m} a_{i} b_{i}=0$, then the inequality $\sum_{i=0}^{m} a_{i} \varphi\left(b_{i}\right) \geq 0$ holds for every real convex function with the nodes, $b_{i}$, in its domain if and only if it holds for

$$
g_{k}(x)=\left\{\begin{array}{cc}
0 & x<b_{k} \\
x-b_{k} & x \geq b_{k}
\end{array}\right.
$$

for $k=0,1, \ldots, m$.

Proof. Since $g_{k}(x)$ are convex functions, we need only to prove the $\Longleftarrow$ part. We can assume that all the nodes are distinct and that $b_{0}<b_{1}<$ $\cdots<b_{m}$. With this additional assumption, it is enough to assume that $\sum_{i=0}^{m} a_{i} g_{k}\left(b_{i}\right) \geq 0$ for $k=1, \ldots, m-1$. Let $\alpha_{0}, \alpha_{1}, \ldots, \alpha_{m-2}$ be from Proposition 1 . Then for each $k=1, \ldots, m-1$.

$$
\begin{aligned}
\sum_{i=0}^{m} a_{i} g_{k}\left(b_{i}\right) & =\sum_{j=0}^{m-2} \alpha_{j}\left[g_{k}: b_{j}, b_{j+1}, b_{j+2}\right] \\
& =\alpha_{k-1}\left[g_{k}: b_{k-1}, b_{k}, b_{k+1}\right]=\alpha_{k-1}\left(b_{k}-b_{k-1}\right)\left(b_{k+1}-b_{k}\right) \geq 0
\end{aligned}
$$

if and only if $\alpha_{k-1} \geq 0$. (The second equality follows from $[c x+d: u, v, w]=$ 0 .) Thus $\alpha_{k} \geq 0$ for $k=0, \ldots, m-2$. Now let $\varphi$ be any real convex function with the nodes in its domain. Then $\sum_{i=0}^{m} a_{i} \varphi\left(b_{i}\right)=\sum_{j=0}^{m-2} \alpha_{j}[\varphi$ : $\left.b_{j}, b_{j+1}, b_{j+2}\right] \geq 0$ since each $\alpha_{j} \geq 0$ and $\varphi$ is convex.

Since $\sum_{i=0}^{m} a_{i} g_{k}\left(b_{i}\right)=\sum_{b_{i}>b_{k}} a_{i}\left(b_{i}-b_{k}\right)$, an immediate consequence of Theorem 2 is the following result.

Corollary 3. If $\sum_{i=0}^{m} a_{i}=0$ and $\sum_{i=0}^{m} a_{i} b_{i}=0$, then $\sum_{i=0}^{m} a_{i} \varphi\left(b_{i}\right) \geq 0$ for every real convex function, $\varphi$, with the nodes in its domain if and only if $\sum_{b_{i}>b_{k}} a_{i}\left(b_{i}-b_{k}\right) \geq 0$ for $k=0,1, \ldots, m$. The inequality $\sum_{b_{i}>b_{k}} a_{i}\left(b_{i}-b_{k}\right) \geq$ 0 can be replaced with $\sum_{b_{i}<b_{k}} a_{i}\left(b_{i}-b_{k}\right) \leq 0$.

The second part follows from the fact that $\sum a_{i}\left(b_{i}-b_{k}\right)=0$.

\section{Proofs of Jensen's and Karamata's inequalities}

In this section we will give proofs of Jensen's and Karamata's inequalities based on our main result.

Corollary 4 (Jensen's inequality). If $\varphi$ is a real convex function defined on $[c, d], x_{1}, x_{2}, \ldots, x_{n}$ in its domain, the weights $a_{i}$ positive, then

$$
\sum_{i=1}^{n} a_{i} \varphi\left(x_{i}\right)-\left(\sum_{j=1}^{n} a_{j}\right) \varphi\left(\frac{\sum_{i=1}^{n} a_{i} x_{i}}{\sum_{j=1}^{n} a_{j}}\right) \geq 0 .
$$


Proof. Let $\bar{x}=\frac{\sum_{i=1}^{n} a_{i} x_{i}}{\sum_{j=1}^{n} a_{j}}$. Since $c \leq x_{i} \leq d$ and $a_{i}>0$, it follows that $c \leq \bar{x} \leq d$. If we write Jensen's inequality in the form $\sum c_{i} \varphi\left(d_{i}\right) \geq 0$, then it is clear that $\sum c_{i}=0$ and $\sum c_{i} d_{i}=0$ so that Corollary 3 applies. Let $z \in\left\{\bar{x}, x_{1}, \ldots, x_{n}\right\}$. If $z \geq \bar{x}$, by Corollary 3 first part we need to check validity of $\sum_{x_{i}>z} a_{i}\left(x_{i}-z\right) \geq 0$ while if $z<\bar{x}$, by the second part of Corollary 3 we need to check validity of $\sum_{x_{i}<z} a_{i}\left(x_{i}-z\right) \leq 0$. Both of these inequalities are true since by assumption each $a_{i}$ is positive.

Before we prove Karamata's inequality we will prove the following lemma on majorized sequences which is interesting in its own right.

Lemma 5. Let $x_{1} \leq x_{2} \leq \cdots \leq x_{n}$ and $y_{1} \leq y_{2} \leq \cdots \leq y_{n}$. If $\sum_{i=r}^{n} y_{i} \leq$ $\sum_{i=r}^{n} x_{i}$ for $r=1,2,3, \ldots, n$, then for every real number $z, \sum_{x_{i} \geq z}\left(x_{i}-z\right) \geq$ $\sum_{y_{i} \geq z}\left(y_{i}-z\right)$.

Proof. The case $z>x_{n}$ is trivial. If $x_{n} \geq z \geq y_{n}$, then $\sum_{x_{i} \geq z}\left(x_{i}-z\right)-$ $\sum_{y_{i}>z}\left(y_{i}-z\right)=\sum_{x_{i} \geq z}\left(x_{i}-z\right) \geq 0$. It remains to verify the case $z<y_{n}$. Let $0 \leq k \leq n-1$ denote the number of $x^{\prime} s$ that are less than or equal $z$, and let $0 \leq r \leq n-1$ denote the number of $y^{\prime} s$ that are less than or equal $z$. Since both sequences are increasing

$$
\sum_{x_{i} \geq z}\left(x_{i}-z\right)-\sum_{y_{i} \geq z}\left(y_{i}-z\right)=\sum_{i=k+1}^{n}\left(x_{i}-z\right)-\sum_{i=r+1}^{n}\left(y_{i}-z\right) .
$$

If $k=r$, then the last equality reduces to $\sum_{i=r+1}^{n} x_{i}-\sum_{i=r+1}^{n} y_{i}$ which is positive by our assumption. If $k>r$, then $\sum_{i=k+1}^{n}\left(x_{i}-z\right)-\sum_{i=r+1}^{n}\left(y_{i}-z\right)$ can be written as $\sum_{i=r+1}^{n}\left(x_{i}-y_{i}\right)-\sum_{i=r+1}^{k}\left(x_{i}-z\right)$. By our assumption $\sum_{i=r+1}^{n}\left(x_{i}-y_{i}\right) \geq 0$, while $\sum_{i=r+1}^{k}\left(x_{i}-z\right) \leq 0$ by the choice of $k$. Thus in this case $\sum_{x_{i} \geq z}\left(x_{i}-z\right) \geq \sum_{y_{i} \geq z}\left(y_{i}-z\right)$. If $k<r$, then $\sum_{i=k+1}^{n}\left(x_{i}-z\right)-$ $\sum_{i=r+1}^{n}\left(y_{i}-z\right)=\sum_{i=r+1}^{n}\left(x_{i}-y_{i}\right)+\sum_{i=k+1}^{r}\left(x_{i}-z\right)$ which again is positive by our assumption and the choice of $k$.

Corollary 6 (Karamata's inequality). Let $x_{1} \leq x_{2} \leq \cdots \leq x_{n}$ and $y_{1} \leq$ $y_{2} \leq \cdots \leq y_{n}$ be in the domain of a real convex function $\varphi$. Suppose that $\sum_{i=k}^{n} y_{i} \leq \sum_{i=k}^{n} x_{i}$ for $k=2,3, \ldots, n$ and $\sum_{i=1}^{n} y_{i}=\sum_{i=1}^{n} x_{i}$. Then $\sum_{i=1}^{n} \varphi\left(x_{i}\right) \geq \sum_{i=1}^{n} \varphi\left(y_{i}\right)$.

Proof. If we write Karamata's inequality $\sum_{i=1}^{n} \varphi\left(x_{i}\right) \geq \sum_{i=1}^{n} \varphi\left(y_{i}\right)$ as $0 \leq$ $\sum_{i=1}^{n} \varphi\left(x_{i}\right)-\sum_{i=1}^{n} \varphi\left(y_{i}\right)=\sum_{i=1}^{2 n} c_{i} \varphi\left(d_{i}\right)$, then clearly $\sum c_{i}=0$ while $\sum c_{i} d_{i}$ $=\sum_{i=1}^{n} x_{i}-\sum_{i=1}^{n} y_{i}=0$ by our assumption. Now by Corollary 3 we have to prove that if $z \in\left\{x_{i}, y_{i}\right\}_{i=1}^{n}$, then $\sum_{x_{i}>z}\left(x_{i}-z\right)-\sum_{y_{i}>z}\left(y_{i}-z\right) \geq 0$. But by Lemma 5 the last inequality is true for any $z$. 
The next result states that these type of inequalities remain true if the nodes are transformed by a linear function.

Corollary 7. Let $c \neq 0$ and $d$ be real numbers. The nodes $\left\{b_{i}\right\}_{i=0}^{m}$ and the corresponding weights $\left\{a_{i}\right\}_{i=0}^{m}$ satisfy $\sum_{i=0}^{m} a_{i} \varphi\left(b_{i}\right) \geq 0$ for every real convex function $\varphi$ with the nodes in its domain, if and only if $\sum_{i=0}^{m} a_{i} \psi\left(c b_{i}+d\right) \geq 0$ for every real convex function $\psi$ with the nodes $\left\{c b_{i}+d\right\}_{i=0}^{m}$ in its domain.

Proof. First suppose that $\sum_{i=0}^{m} a_{i} \varphi\left(b_{i}\right) \geq 0$ for every real convex function. As was pointed out earlier this assumption implies that $\sum_{i=0}^{m} a_{i}=0$ and $\sum_{i=0}^{m} a_{i} b_{i}=0$. Consequently $\sum_{i=0}^{m} a_{i}\left(c b_{i}+d\right)=0$. Assume that for each $i=0,1, \ldots, m, c b_{i}+d$ is in the domain of a convex function $\psi$. By Corollary 3 we have to verify the inequality $\sum_{c b_{j}+d>c b_{k}+d} a_{j}\left(\left(c b_{j}+d\right)-\left(c b_{k}+d\right)\right) \geq 0$ for each $k=0,1, \ldots, m$. We have

$$
\sum_{c b_{j}+d>c b_{k}+d} a_{j} c\left(b_{j}-b_{k}\right)=\left\{\begin{array}{ll}
c \sum_{b_{j}>b_{k}} a_{j}\left(b_{j}-b_{k}\right) & \text { if } c \geq 0 \\
c \sum_{b_{j}<b_{k}} a_{j}\left(b_{j}-b_{k}\right) & \text { if } c<0
\end{array} \geq 0\right.
$$

again by Corollary 3 applied to $\varphi$. The converse follows from this case applied to the pair $\frac{1}{c}$ and $\frac{-d}{c}$.

\section{Nodes Whose Differences ARE INTEGERS}

If $b_{i}-b_{0}$ is an integer for each $i=1,2, \ldots, m$, then for any function $f$ we can write $\sum_{i=0}^{m} a_{i} f\left(b_{i}\right)=\sum_{j=0}^{R} a_{j} f\left(b_{0}+j\right)$ where $R=b_{m}-b_{0}$, and $a_{j}=\left\{\begin{array}{cc}a_{i} & \text { if } b_{0}+j=b_{i} \\ 0 & \text { otherwise }\end{array}\right.$. If in addition, $b_{0}<b_{1}<\cdots<b_{m}, \sum_{i=0}^{m} a_{i}=0$ and $\sum_{i=0}^{m} a_{i} b_{i}=0$, the statement of Proposition 1, for the function $f(t)=x^{t}$, takes on the simple form

$$
\begin{aligned}
& \sum_{i=0}^{m} a_{i} x^{b_{i}}=\sum_{i=0}^{m} a_{i} f\left(b_{i}\right)=\sum_{j=0}^{R} a_{j} f\left(b_{0}+j\right) \\
& \quad=\sum_{j=0}^{R-2} \alpha_{j}\left[x^{t}: b_{0}+j, b_{0}+j+1, b_{0}+j+2\right]=x^{b_{0}}(x-1)^{2}\left(\sum_{j=0}^{R-2} \alpha_{j} x^{j}\right)
\end{aligned}
$$

where the last equality follows from $\left[x^{t}: u, u+1, u+2\right]=x^{u}\left(x^{2}-1\right)$. Recall that the numbers $\alpha_{j}$ from the statement of Proposition 1 depend only on the nodes and the weights. In particular if $\varphi$ is any convex function defined on $[c, d]$ that contains all of the nodes, then for the same $\alpha_{j}$ s we also have

$$
\sum_{i=0}^{m} a_{i} \varphi\left(b_{i}\right)=\sum_{j=0}^{R-2} \alpha_{j}\left[\varphi: b_{0}+j, b_{0}+j+1, b_{0}+j+2\right] .
$$


Now it is easy to modify the proof of Theorem 2 to obtain our next result.

Theorem 8. Suppose that the nodes are integers, $b_{0}$ the smallest of them and $\left\{b_{0}, b_{1}, \ldots, b_{m}\right\} \subseteq[p, q]$. Then $\sum_{i=0}^{m} a_{i} \varphi\left(b_{i}\right) \geq 0$ for every real convex function $\varphi$ defined on $[p, q]$ if and only if $x^{-b_{0}} \sum_{i=0}^{m} a_{i} x^{b_{i}} /(x-1)^{2}$ is a polynomial with positive coefficients.

Proof. First notice that if $h(x)=x^{-b_{0}} \sum_{i=0}^{m} a_{i} x^{b_{i}}$, then $\sum_{i=0}^{m} a_{i}=0$ and $\sum_{i=0}^{m} a_{i} b_{i}=0$ if and only if $h(1)=h^{\prime}(1)=0$ if and only if $h(x) /(x-1)^{2}$ is a polynomial. Thus under either condition $\sum_{i=0}^{m} a_{i} \varphi\left(b_{i}\right) \geq 0$ for every real convex function $\varphi$ or $x^{-b_{0}} \sum_{i=0}^{m} a_{i} x^{b_{i}} /(x-1)^{2}$ is a polynomial, we have $\sum_{i=0}^{m} a_{i}=0$ and $\sum_{i=0}^{m} a_{i} b_{i}=0$. As in the proof of Theorem 2 we may assume that $b_{0}<b_{1}<\cdots<b_{m}$. By Proposition 1 there are numbers $\alpha_{0}, \alpha_{1}, \ldots, \alpha_{R-2}$ such that

$$
\sum_{i=0}^{m} a_{i} \varphi\left(b_{i}\right)=\sum_{j=0}^{R-2} \alpha_{j}\left[\varphi: b_{0}+j, b_{0}+j+1, b_{0}+j+2\right]
$$

and

$$
\sum_{i=0}^{m} a_{i} x^{b_{i}}=x^{b_{0}}(x-1)^{2}\left(\sum_{j=0}^{R-2} \alpha_{j} x^{j}\right) .
$$

Suppose each $\alpha_{j}$ is positive and let $\varphi$ be convex. Then for each $j=$ $0,1, \ldots, R-2,\left[\varphi: b_{0}+j, b_{0}+j+1, b_{0}+j+2\right] \geq 0$ and hence each term of the first equation (2) is positive. Thus $\sum_{i=0}^{m} a_{i} \varphi\left(b_{i}\right) \geq 0$. This proves the $\Longleftarrow$ part. To prove the $\Longrightarrow$ part, for $1 \leq k \leq R-1$, we consider the convex functions

$$
\varphi(x)=g_{k}(x)=\left\{\begin{array}{cc}
0 & x<b_{0}+k \\
x-\left(b_{0}+k\right) & x \geq b_{0}+k
\end{array} .\right.
$$

As in the proof of Theorem 2 the sum

$$
\sum_{i=0}^{m} a_{i} \varphi\left(b_{i}\right)=\sum_{j=0}^{R-2} \alpha_{j}\left[\varphi: b_{0}+j, b_{0}+j+1, b_{0}+j+2\right]
$$

reduces to

$$
\alpha_{k-1}\left[\varphi: b_{0}+k-1, b_{0}+k, b_{0}+k+1\right]=\alpha_{k-1} \geq 0 .
$$

Thus $\alpha_{0}, \alpha_{1}, \ldots, \alpha_{R-2}$ are all positive, and hence from (3) it follows that $x^{-b_{0}} \sum_{i=0}^{m} a_{i} x^{b_{i}} /(x-1)^{2}$ is a polynomial with positive coefficients.

If there is an $h$ such that $b_{k}-b_{0}$ is an integer multiple of $h$, (which is the case if all the nodes are rational numbers,) then Corollary 7 and Theorem 8 produce the following result. 
Corollary 9. Suppose there is an $h$ such that for $b_{0}<b_{1}<\cdots<b_{m}$ we have $b_{k}-b_{0}$ is an integer multiple of $h$ for $k=1, \ldots, m$. Then $\sum_{i=0}^{m} a_{i} \varphi\left(b_{i}\right) \geq 0$ for every real convex function $\varphi$ defined on $\left[b_{0}, b_{m}\right]$ if and only if $\sum_{i=0}^{m} a_{i} x^{\frac{b_{i}-b_{0}}{h}} /$ $(x-1)^{2}$ is a polynomial with positive coefficients.

Proof. First we apply Corollary 7 with $c=\frac{1}{h}$ and $d=-\frac{b_{0}}{h}$. Thus $\sum_{i=0}^{m} a_{i} \varphi\left(b_{i}\right)$ $\geq 0$ for every real convex function $\varphi$ defined on $\left[b_{0}, b_{m}\right]$ if and only if $\sum_{i=0}^{m} a_{i} \psi\left(\frac{b_{i}-b_{0}}{h}\right) \geq 0$ for every real convex function $\psi$ defined on $\left[0, \frac{b_{m}-b_{0}}{h}\right]$. Since by the assumption $\frac{b_{i}-b_{0}}{h}$ are integers, from Theorem 8 we obtain that $\sum_{i=0}^{m} a_{i} \psi\left(\frac{b_{i}-b_{0}}{h}\right) \geq 0$ for every real convex function defined on $\left[0, \frac{b_{m}-b_{0}}{h}\right]$ if and only if $\sum_{i=0}^{m} a_{i} x^{\frac{b_{i}-b_{0}}{h}} /(x-1)^{2}$ is a polynomial with positive coefficients.

We finish this section with another application of Theorem 8. For every real convex function $\varphi$ on real line

$$
43 \varphi(5)-82 \varphi(4)+63 \varphi(3)-51 \varphi(2)+26 \varphi(1)+\varphi(0) \geq 0 .
$$

This can be verified by Karamata's inequality but it is much easier to apply Theorem 8. All we need to check is that

$$
\left(43 x^{5}-82 x^{4}+63 x^{3}-51 x^{2}+26 x+1\right) /(x-1)^{2}
$$

is a polynomial with all positive coefficients. Indeed

$$
43 x^{5}-82 x^{4}+63 x^{3}-51 x^{2}+26 x+1=(x-1)^{2}\left(1+28 x+4 x^{2}+43 x^{3}\right) .
$$

On the other hand it is not true that

$$
43 \varphi(5)-87 \varphi(4)+73 \varphi(3)-56 \varphi(2)+26 \varphi(1)+\varphi(0) \geq 0
$$

for every convex function because this time

$$
43 x^{5}-87 x^{4}+73 x^{3}-56 x^{2}+26 x+1=(x-1)^{2}\left(1+28 x-x^{2}+43 x^{3}\right) .
$$

\section{INEQUALITIES FOR $n$ CONVEX FUNCTIONS}

In this section we briefly discuss inequalities for $n$ convex functions. Convexity can be described via divided differences. If $u, v$, and $w$ are three distinct points, then $[u, v, w: f]=\frac{f(u)}{(u-v)(u-w)}+\frac{f(v)}{(v-u)(v-w)}+\frac{f(w)}{(w-u)(w-v)}$ is called the divided difference of $f$ at points $u, v$, and $w$. A function is convex if and only if $[u, v, w: f] \geq 0$ for any three distinct points $u, v$, and $w$ from its domain. If we consider a set $V$ of $n+1$ distinct points, then we say that $f$ is $n$ convex if $[V: f]=\sum_{u \in V} \frac{f(u)}{\prod_{v \neq u}(u-v)} \geq 0$ for any such set $V$ from the domain of $f$. Thus being convex is equivalent to being 2 convex. One can see that increasing and 1 convex are equivalent concepts and the same is true for nonnegative and 0 convex. An interested reader can find more 
information about $n$ convex functions in [2]. Proposition 1 was instrumental in obtaining our results for convex functions. For $n$ convex functions this proposition takes on the following form.

Proposition 10. Suppose that $\sum_{i=0}^{m} a_{i} b_{i}^{k}=0$ for $k=0,1, \ldots, n-1$. If $b_{0}<b_{1}<\cdots<b_{m}$, then there are numbers $\alpha_{0}, \ldots, \alpha_{m-n}$ such that for every function $f$ we have $\sum_{i=0}^{m} a_{i} f\left(b_{i}\right)=\sum_{j=0}^{m-n} \alpha_{j}\left[b_{j}, b_{j+1}, \ldots, b_{j+n}: f\right]$.

Now in the case of increasing functions (the case $n=1$ ) the role of the functions $g_{k}(x)$ in the statement of Theorem 2 are played by increasing functions $g_{k}(x)=\left\{\begin{array}{ll}0 & x<b_{k} \\ 1 & x \geq b_{k}\end{array}\right.$ and Corollary 3 takes on the following form.

Theorem 11. Suppose that $\sum_{i=0}^{m} a_{i}=0$. If $b_{0} \leq b_{1} \leq \cdots \leq b_{m}$; then $\sum_{i=0}^{m} a_{i} f\left(b_{i}\right) \geq 0$ for every increasing function $f$ if and only if $\sum_{i=k}^{m} a_{i} \geq 0$ for $k=1,2, \ldots, m$.

Unfortunately the inequalities for $n$ convex for $n \geq 3$ are not as nice as those for convex functions. For example in the case of integer nodes only the easy implication of Theorem 8 is true.

Theorem 12. Let $m, n$ be integers with $m \geq n+1$. Suppose that $p=$ $b_{0}<b_{1}<\cdots<b_{m}=q$ are integers. If $x^{-p} \sum_{i=0}^{m} a_{i} x^{b_{i}} /(x-1)^{n}$ is a polynomial with positive coefficients, then $\sum_{i=0}^{m} a_{i} \varphi\left(b_{i}\right) \geq 0$ for every real $n$ convex function $\varphi$ defined on $[p, q]$.

We omit the proofs since they are very similar to the proofs of the corresponding results for convex functions. But the converse fails for $n \geq 3$ as the following example shows. We will show that

$$
-5 f(0)+16 f(1)-22 f(2)+20 f(3)-13 f(4)+4 f(5) \geq 0
$$

for every 3 convex function defined on $[0,5]$. Since

$$
-5+16 x-22 x^{2}+20 x^{3}-13 x^{4}+4 x^{5}=(x-1)^{3}\left(5-x+4 x^{2}\right)
$$

this will be a counterexample to the converse of Theorem 12. Let $g(x)=$ $f(x / 2)$; then $g$ is 3 convex on $[0,10]$ and

$$
-5 g(0)+16 g(2)-22 g(4)+20 g(6)-13 g(8)+4 g(10) \geq 0
$$

since by Theorem 12 the corresponding polynomial

$$
\begin{aligned}
-5 & +16 x^{2}-22 x^{4}+20 x^{6}-13 x^{8}+4 x^{10}=\left(x^{2}-1\right)^{3}\left(5-x^{2}+4 x^{4}\right) \\
& =(x-1)^{3}\left(5+15 x+14 x^{2}+2 x^{3}+x^{4}+11 x^{5}+12 x^{6}+4 x^{7}\right) .
\end{aligned}
$$

Now the inequality (4) follows from (5). 


\section{REFERENCES}

[1] Z. Kadelburg, D. Djukić, M. Lukić and I. Matić, Inequalities of Karamata, Schur and Muirhead and some applications, The Teaching of Mathematics, 8 (1) (2005), 31-45.

[2] H. Fejzić, R. Svetic and C. E. Weil, Differentiation of $n$ convex functions, Fundam. Math., 209 (2010), 9-25.

(Received: September 24, 2012)

(Revised: December 29, 2012)
Hajrudin Fejzić

Department of Mathematics

California State University

San Bernardino, CA 92407-2397

U. S. A.

hfejzic@csusb.edu

Fuad Živojević

Sarajevo, Bosnia and Herzegovina

fuad.zivojevic@adsfbih.gov.ba 